\title{
Colorectal cancer combination therapy using drug and gene co-delivered, targeted poly(ethylene glycol)- $\varepsilon$-poly(caprolactone) nanocarriers
}

This article was published in the following Dove Press journal:

Drug Design, Development and Therapy

\section{Zhiyu Wang \\ Yaning Wei \\ Guotao Fang \\ Dan Hong \\ Lin An \\ Ting Jiao \\ Yan Shi \\ Aimin Zang}

Hebei Key Laboratory of Cancer Radiotherapy and Chemotherapy, Department of Medical Oncology, Affiliated Hospital of Hebei University, Baoding 071000, People's Republic of China
Correspondence: Yaning Wei

Department of Medical Oncology, Affiliated Hospital of Hebei University,

No 648 Dongfeng East Road, Lanchi

District, Baoding, Baoding 071000, Hebei

Province, People's Republic of China

Tel +86 3 I2 5983042

Email weiynhebu@I63.com
Purpose: Combination therapy is a promising strategy to treat cancer due to the synergistic effects. The drug and gene co-delivered systems attract more attention in the field of combination therapy.

Materials and methods: In the present research, poly(ethylene glycol)- $\varepsilon$-poly(caprolactone) block copolymer was used for the co-loading of 5-fluorouracil (5-FU) and gene. The physicochemical characteristics, in vitro and in vivo anticancer, and gene transfection efficiency were tested on colon cancer cells and tumor-bearing mice.

Results: 5-FU and gene co-loaded nanocarriers had a size of $145 \mathrm{~nm}$. In vivo gene delivery results showed about $60 \%$ of gene-positive cells. Tumor volume of nanocarrier groups at day 21 was around $320 \mathrm{~mm}^{3}$, which is significantly smaller compared with free 5-FU group $\left(852 \mathrm{~mm}^{3}\right)$ and control group $\left(1,059 \mathrm{~mm}^{3}\right)$. The maximum 5-FU plasma concentration in nanocarrier groups (49 $\mu \mathrm{g} / \mathrm{mL})$ was significantly greater than free 5 -FU $(13 \mu \mathrm{g} / \mathrm{mL})$. At 24 hours, drug level of nanocarrier groups was about $2.8 \mu \mathrm{g} / \mathrm{mL}$ compared with $0.02 \mu \mathrm{g} / \mathrm{mL}$ of free 5 -FU.

Conclusion: The resulting nanocarriers co-loaded with the anticancer drugs and genes could be considered as a promising nanomedicine for colorectal cancer therapy.

Keywords: colorectal cancer, gene therapy, combination therapy, cytotoxicity, transfection efficiency

\section{Introduction}

Colorectal cancer is the third most frequently occurring cancer and a common cause of cancer-related death worldwide. ${ }^{1-3}$ Currently, besides the surgical removal, both conventional (chemotherapy and radiotherapy) and innovative approaches (personalized single-targeted therapies) are used for the cure of this type of cancer. ${ }^{4}$ However, significant side effects were often introduced by these therapies, ${ }^{5}$ and drug resistance may have influences on their long-term effectiveness. ${ }^{6}$ Conventional chemotherapy may have adverse effects on the body as it cannot deliver selective action specifically to the cancer cells, thus bring toxicity to healthy cells. ${ }^{7}$ Gene therapy, which holds great promise in treating inherited and acquired diseases, may be an alternative strategy. ${ }^{8}$ For example, Yang et al demonstrated that macrophage inhibitory cytokine-1 (MIC-1) and its associated signals determined the colon cancer cell response to the chemical ribotoxic stress. ${ }^{9}$ Both promoter activity and mRNA stability of MIC-1 gene were upregulated by ribotoxic anisomycin via the p38 MAP kinase signaling pathway.

Combination therapy is a promising strategy for synergistic anticancer treatment. ${ }^{10}$ It has different mechanisms of action that could reduce the dose of each agent, thus may reduce the individual drug-related toxicity. The drug and gene co-delivered systems 
attract more attention in the field of combination therapy. ${ }^{11}$ However, the differences in the physicochemical properties of drug and gene bring about challenges to the systems. ${ }^{12}$ Non-viral nanosystems such as polyplexes are widely used for DNA and drug combination therapy. ${ }^{13,14}$ These systems are constructed by the electrostatic interactions between the polymer, drug, and DNA.

One of the most important tools in cell biology is green fluorescent protein (GFP) from Aequorea victoria. ${ }^{15}$ Enhanced GFP (EGFP) is one of the first engineered versions of GFP. ${ }^{16}$ EGFP could increase the fluorescence due to a higher proportion of correctly folded protein. ${ }^{17}$ It has a single excitation peak at $490 \mathrm{~nm}$ and has been codon optimized for expression in mammalian hosts. So plasmid encoding EGFP (pEGFP) was used as a model gene. For example, Kong et al designed a mannosylated polyethylene glycol (PEG)phosphatidylethanolamine ligand to achieve active targeted nanocarriers (NCs) for the delivery of pEGFP. ${ }^{18} \mathrm{Yu}$ et al developed a cationic solid lipid nanoparticles containing a synthesized single-tailed lipid as a modifier for $\mathrm{pEGFP}$ delivery to treat lung cancer. ${ }^{19}$

5-Fluorouracil (5-FU) is an equivalent of uracil, in which the hydrogen atom at the $\mathrm{C}-5$ position is replaced by a fluorine atom, that promptly enters into the cells by means of the uracil transport mechanism. ${ }^{20}$ 5-FU has been widely applied in diverse severe cancer therapy, including colorectal cancer. Kamel et al prepared chitosan-coated cinnamon/oreganoloaded solid lipid nanoparticles to augment 5-FU cytotoxicity for colorectal cancer. ${ }^{21}$ Pretel et al utilized nanoprecipitation and solvent evaporation methods to optimize poly(D,Llactide-co-glycolide) nanoparticles for the delivery of 5-FU. ${ }^{22}$ In Sharma et al's investigation, 5-FU was conjugated to PEGanchored recombinant human serum albumin nanoparticles to improve the pharmacokinetics and therapeutic profiles. ${ }^{23}$ These NCs can optimize the antitumor activity of 5-FU, thus being a potential nanotool against colon cancer.

In the present study, poly(ethylene glycol)- $\varepsilon$ poly(caprolactone) block copolymer (PEG-PCL) was used for the co-loading of 5-FU and pEGFP. The physicochemical characteristics, in vitro and in vivo anticancer and gene transfection efficiency, were tested on colon cancer cells and tumor-bearing mice. This system was expected to achieve high loading capacity, enhance the therapeutic efficacy, and reduce the side effects.

\section{Materials and methods}

Chemicals and reagents pEGFP-N1 was obtained from Solarbio Life Sciences (Beijing, China). 5-FU ( $\geq 99 \%$ ) and didecyldimethylammonium bromide (98\%) were purchased from Sigma-Aldrich China (Shanghai, China). PEG-PCL (molecular weight 5-5.5 kDa) was purchased from Polymer Source (Montreal, QC, Canada). DMEM, FBS, penicillin/ streptomycin, and MTT were purchased from Thermo Fisher Scientific (Waltham, MA, USA). Quant-iT ${ }^{\mathrm{TM}}$ PicoGreen $^{\circledR}$ dsDNA quantitation reagent was obtained from Thermo Fisher Scientific.

\section{Preparation of NCs, drug encapsulation, and gene loading}

About $100 \mathrm{mg}$ of PEG-PCL was dissolved in $10 \mathrm{~mL}$ ethanol and then mixed with $10 \mathrm{mg}$ of 5-FU (dissolved in $1 \mathrm{~mL}$ ethanol) and dimethyldioctadecylammonium bromide (1\%). ${ }^{24} \mathrm{~A}$ thin film was created by evaporating the mixed solution at reduced pressure. The film was then reconstituted in water and sonicated for 5 minutes, centrifuged at 10,000 rpm for 5 minutes to form 5-FU-loaded nanocarriers (FNCs). Empty nanocarriers (ENCs) were prepared by the same way without adding 5-FU. pEGFP (DNA) was loaded to the ENCs by adding equal volume of ENCs solution rapidly to the DNA (80 $\mathrm{mg} / \mathrm{mL}$ ) and mixed by vortex followed by 30 minutes incubation at room temperature to get DNA-loaded NCs (DNCs). ${ }^{25}$ DNA and 5-FU co-loaded NCs (DFNCs) were achieved by the same way using FNCs instead of ENCs (Figure 1). The above prepared supernatants containing NCs were removed and collected by freeze-drying.

\section{Particle characterization and serum stability}

The hydrodynamic size, polydispersity, and zeta potential values of NCs were measured using dynamic light scattering (DLS) and laser Doppler methods by ELS-Z (Otsuka Electronics Co., Ltd., Hirakata, Japan) according to the manufacturer's instructions. ${ }^{26}$ To investigate the stability of NCs in serum, NC suspension was mixed with FBS (1:1, volume:volume) and analyzed by DLS method.

\section{Drug and gene entrapment efficiency (DEE and GEE) and release behavior}

The DEE of 5-FU in NCs was quantified by high-performance liquid chromatography (LC-20A; Shimadzu, Tokyo, Japan) using $250 \times 4.6 \mathrm{~mm} \mathrm{C1} 8$ column. ${ }^{27}$ The mobile phase $(0.05 \mathrm{M}$ of $\mathrm{KH}_{2} \mathrm{PO}_{4}$ contained $0.1 \%$ of triethylamine) has a flow rate of $0.6 \mathrm{~mL} / \mathrm{min}$, and ultraviolet detection was set at $266 \mathrm{~nm}$. The DEE (\%) was calculated according to the equation: (weight of 5-FU in $\mathrm{NCs} /$ weight of total 5-FU fed) $\times 100$. 


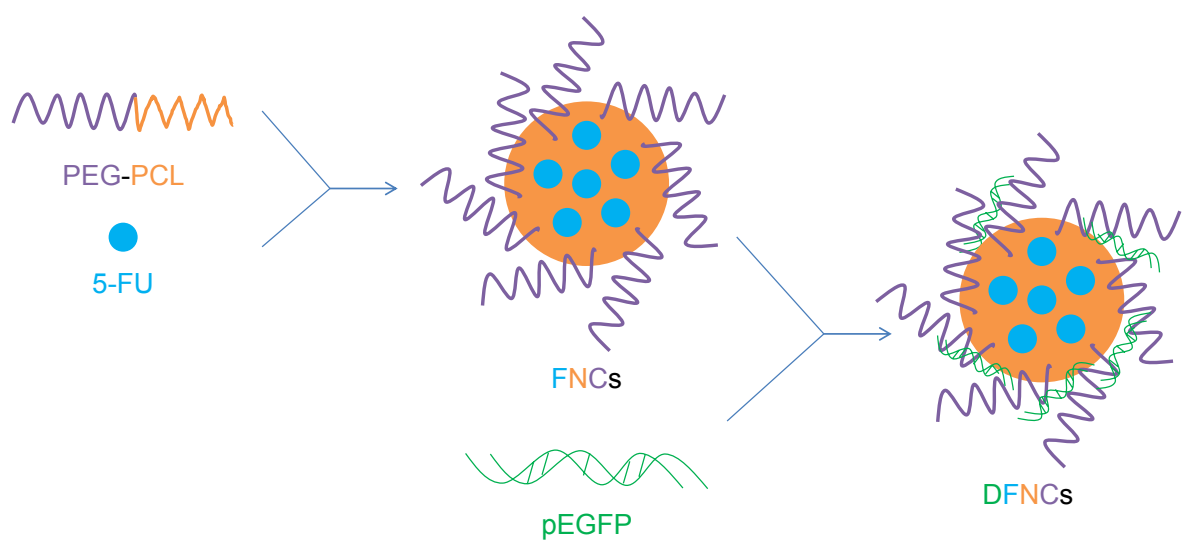

Figure I Preparation of DNA and 5-FU co-loaded NCs.

Abbreviations: NC, nanocarrier; FNC, 5-FU-loaded nanocarrier; DFNC, DNA and 5-FU co-loaded nanocarrier; 5-FU, 5-fluorouracil; PEG-PCL, poly(ethylene glycol)-Epoly(caprolactone) block copolymer; pEGFP, plasmid encoding enhanced green fluorescent protein.

GEE of NCs was determined by PicoGreen ${ }^{\circledR}$ fluorometry assay. ${ }^{28}$ It was calculated according to the linear calibration curve of pEGFP, according to the equation: (weight of total pEGFP - free $\mathrm{pEGFP}) /($ weight of total pEGFP $) \times 100$.

Release of 5-FU and DNA from NCs was performed in PBS (pH 7.4). ${ }^{29}$ Aliquots of NCs (equivalent to $2 \mu \mathrm{g}$ DNA) were suspended in Eppendorf ${ }^{\circledR}$ tubes containing $1 \mathrm{~mL}$ of PBS and vortexed. Separate tubes were used for each data point. The tubes were then placed in a shaking water bath $\left(37^{\circ} \mathrm{C}\right.$, $100 \mathrm{rpm})$. At predetermined time intervals, the suspensions were centrifuged (15,000 rpm, 20 minutes), and the amount of 5-FU and DNA released in the NCs was analyzed by the same way above.

\section{Cell culture}

Caco-2 (ATCC ${ }^{\circledR}$ HTB-37'M , human colon adenocarcinoma) and SW480 (ATCC ${ }^{\circledR}$ CCL-228 ${ }^{\mathrm{TM}}$, human Dukes' type B, colorectal adenocarcinoma) cells were purchased from American Type Culture Collection (Manassas, VA, USA). Cells were cultured in DMEM containing $10 \%$ FBS, $1 \%$ nonessential amino acids, $100 \mathrm{U} / \mathrm{mL}$ penicillin, and $0.1 \mathrm{mg} / \mathrm{mL}$ streptomycin at $37^{\circ} \mathrm{C}$ in the atmosphere of $5 \% \mathrm{CO}_{2}$ and $95 \%$ relative humidity.

\section{In vitro cytotoxicity test}

The cytotoxicity of Caco-2 and SW480 cells treated with NCs were evaluated by using MTT assay. ${ }^{30} \mathrm{Cells}$ were seeded into 96-well microplates at a concentration of $10^{5}$ cells $/ \mathrm{mL}$ and allowed to grow for 24 hours to a subconfluent state ( $80 \%$ confluence). The culture medium was replaced with fresh medium supplemented with $10 \%$ FBS having different concentrations of free 5-FU (5-FU) or different kinds of $\mathrm{NCs}$, and samples were incubated for 72 hours with $100 \mu \mathrm{L}$ of tested suspensions. Then suspensions were removed and $50 \mu \mathrm{L}$ of MTT solution ( $1 \mathrm{mg} / \mathrm{mL}$ in culture medium) was added and cells were incubated for 4 hours at $37^{\circ} \mathrm{C}$ in $5 \% \mathrm{CO}_{2}$ prior to the analysis. Thereafter, the medium was removed and $100 \mu \mathrm{L}$ of dimethyl sulfoxide was added to dissolve blue formazan crystals. The absorbance of the formed dye was measured at $570 \mathrm{~nm}$ using a microplate reader. Absorbance values for untreated cells were taken as control $(100 \%$ survival). Cell viability was calculated according to the equation: (absorbance of test cells)/(absorbance of control) $\times 100$.

\section{Mouse model}

BALB/c nude mice (6-8 weeks old) were purchased from Beijing Vital River Experimental Animal Technical Co., Ltd (Beijing, China). About $10^{6}$ of SW480 cells was suspended in $100 \mu \mathrm{L} 0.9 \%$ saline and injected to the dorsal side of the mice to induce the colorectal cancer-bearing mice. Tumors were allowed to reach 4-5 $\mathrm{mm}$ in diameter before the initiation of the experiments. All the animal experiments were approved by the Medical Ethics Committee of Hebei University (No 20201800113001) and followed the National Institutes of Health guide for the care and use of laboratory animals (NIH Publications No 8023, revised 1978).

\section{In vivo gene transfection efficiency}

In vivo gene transfection efficiency of NCs was evaluated on colorectal cancer-bearing mice. ${ }^{31}$ Five groups of tumor-bearing mice (eight per group) were injected intravenously with naked pEGFP (DNA), ENCs, FNCs, DNCs, and DFNCs. The DNA released from the NCs was over $70 \%$ at 24 hours and nearly $90 \%$ at 72 hours. So at 24 or 72 hours post injection, mice were sacrificed. The tumor tissues were taken out and homogenized by pressing the samples through a $30-\mathrm{mm}$ cell mesh with the 
plunger of a 10-mL syringe. Erythrocyte lysis buffer was added during homogenization to lyse the red blood cells, washed three times with PBS containing $0.5 \%$ bovine serum albumin, and then filtered. After centrifugation at $4^{\circ} \mathrm{C}$ and $1,000 \mathrm{rpm}$ for 5 minutes, the cells were seeded into 24-well plates in $1 \mathrm{~mL}$ of DMEM with 10\% FBS. An inversion fluorescence microscope was used to observe the fluorescent cells and the pictures were taken. Then the cells were detached with trypsin/EDTA. To quantify the amount of cells that were successfully transfected, the supernatant was discarded and resuspended with $300 \mathrm{~mL}$ of PBS and added into the flow cytometry.

\section{In vivo anticancer activity}

Colorectal cancer-bearing mice were divided to six groups (eight per group) and were injected intravenously with $20 \mathrm{mg} / \mathrm{kg}$ of $0.9 \%$ sodium chloride solution (blank control), free 5-FU, ENCs, FNCs, DNCs, and DFNCs. ${ }^{32}$ All samples were diluted with $0.9 \%$ sodium chloride and injected every 3 days. Following drug administration, body weight and tumor growth were measured every 3 days. The tumor volume $\left(\mathrm{mm}^{3}\right)$ was calculated according to the equation: (length $\times$ width $)^{2} / 2$.

On the 18th day after the first administration, the mice were killed and the tumor of each mouse was weighed, and tumor inhibition ratios (\%) were calculated according to the equation: (tumor weight of the control - tumor weight of the treated)/(tumor weight of the control $) \times 100$.

\section{In vivo pharmacokinetic study}

Colorectal cancer-bearing mice were divided to three groups (eight per group) and were injected intravenously with a single dose of free 5-FU, FNCs, and DFNCs (10 mg 5-FU/kg). ${ }^{33}$ Mice were euthanized and blood sample was collected at determined time points. Blood samples were centrifuged at 25,000 rpm for 15 minutes to separate the plasma. Plasma concentrations of 5-FU were determined using the same way as in Drug and gene entrapment efficiency (DEE and GEE) and release behavior Section.

\section{Statistical analysis}

Statistical analysis was performed using Student's $t$-test. Differences between groups were considered significant at $* P<0.05$. All statistical analyses were performed using SPSS version 19.0 package, and the values were expressed as mean \pm standard error of the mean.

\section{Results \\ NC characterization}

The average hydrodynamic size of ENCs and FNCs was around $110 \mathrm{~nm}$ while the DNA-entrapped DNCs and DFNCs had sizes of about $145 \mathrm{~nm}$ (Table 1). The size distribution seemed to be narrow considering its polydispersity index which is lower than 0.2 . The reason for narrow size distribution may be the thin firm sonication method used in this study. The DEE of 5-FU in NCs was above $80 \%$ and GEE was $\sim 90 \%$. NCs exhibited no obvious changes in the hydrodynamic size and polydispersity after mixing with serum media (Figure 2). The NCs may be stable in the presence of serum when administrated in vivo.

\section{Drug and gene release behavior}

Cumulative 5-FU and DNA release profiles of FNCs, DNCs, and DFNCs were calculated in Figure 3. The release of DNA from NCs was faster than 5 -FU, over $80 \%$ of release were found at 48 and 72 hours for DNA and drug, respectively. DNA release behaviors of DNCs and DFNCs are similar. The release of drug from FNCs was a bit faster than that from DFNCs. 5-FU released from DFNCs was slower at the first 24 hours and then faster after 24 hours until complete release.

\section{In vitro cytotoxicity}

Free 5-FU, which has been used as a chemotherapeutic agent, exhibited antiproliferation activities in Caco-2 and SW480 cells. As shown in Figure 4, there was no significant cytotoxicity of ENC and DNC (without drug) groups at the studied concentrations. However, 5-FU-containing groups showed cytotoxicity in a dose-dependent manner. 5-FU-loaded NCs

Table I NC characterizations (mean \pm standard error, $\mathrm{n}=3$ )

\begin{tabular}{llllll}
\hline NCs & $\begin{array}{l}\text { Hydrodynamic } \\
\text { size }(\mathbf{n m})\end{array}$ & PDI & $\begin{array}{l}\text { Zeta potential } \\
(\mathbf{m V})\end{array}$ & DEE (\%) & GEE (\%) \\
\hline ENCs & $116.7 \pm 3.9$ & $0.122 \pm 0.019$ & $+27.6 \pm 2.9$ & N/A & N/A \\
FNCs & $113.9 \pm 4.5$ & $0.139 \pm 0.025$ & $+25.9 \pm 3.1$ & $83.9 \pm 3.6$ & N/A \\
DNCs & $145.5 \pm 5.2$ & $0.159 \pm 0.031$ & $+16.3 \pm 2.6$ & $81.7 \pm 4.2$ & $90.7 \pm 4.5$ \\
DFNCs & $148.3 \pm 5.7$ & $0.171 \pm 0.039$ & $+15.4 \pm 3.2$ & \\
\hline
\end{tabular}

Abbreviations: 5-FU, 5-fluorouracil; DEE, drug entrapment efficiency; DFNC, DNA and 5-FU co-loaded nanocarrier; DNC, DNA-loaded nanocarrier; ENC, empty nanocarrier; FNC, 5-FU-loaded nanocarrier; GEE, gene entrapment efficiency; NC, nanocarrier; PDI, polydispersity index. 

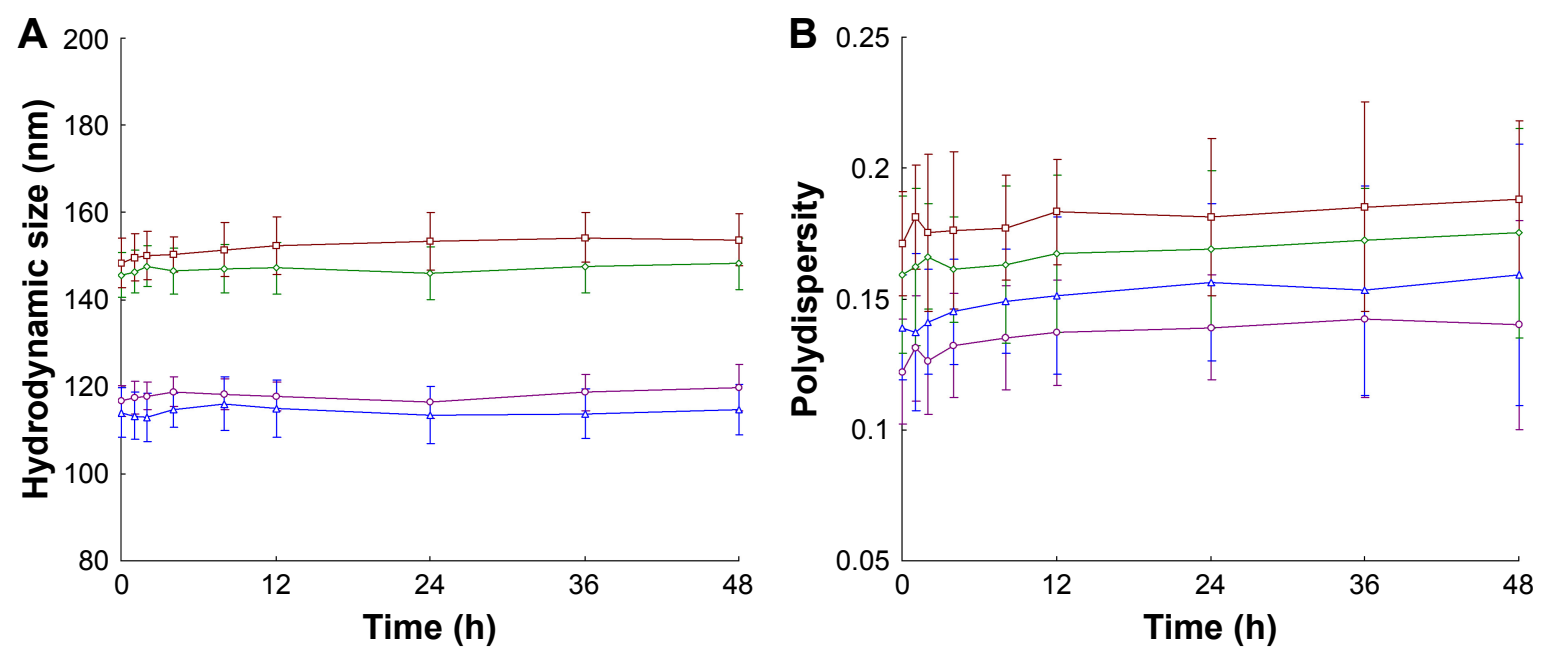

$\multimap-$ ENCs $\rightarrow$ FNCs $\rightarrow$ DNCs $\rightarrow-$ DFNCs

Figure 2 Changes in the hydrodynamic size (A) and polydispersity (B) after mixing with serum media.

Note: Data are presented as means \pm standard error, $n=3$.

Abbreviations: 5-FU, 5-fluorouracil; DFNC, DNA and 5-FU co-loaded nanocarrier; DNC, DNA-loaded nanocarrier; ENC, empty nanocarrier; FNC, 5-FU-loaded nanocarrier.

achieved significantly more inhibition efficiency in colon cancer cells compared with free 5-FU $(P<0.05)$.

\section{In vivo gene transfection}

In vivo gene transfection activity of DNCs and DFNCs was evaluated in vivo using DNA, ENCs, and FNCs as contrast. Figure 5A exhibited the qualitative examination

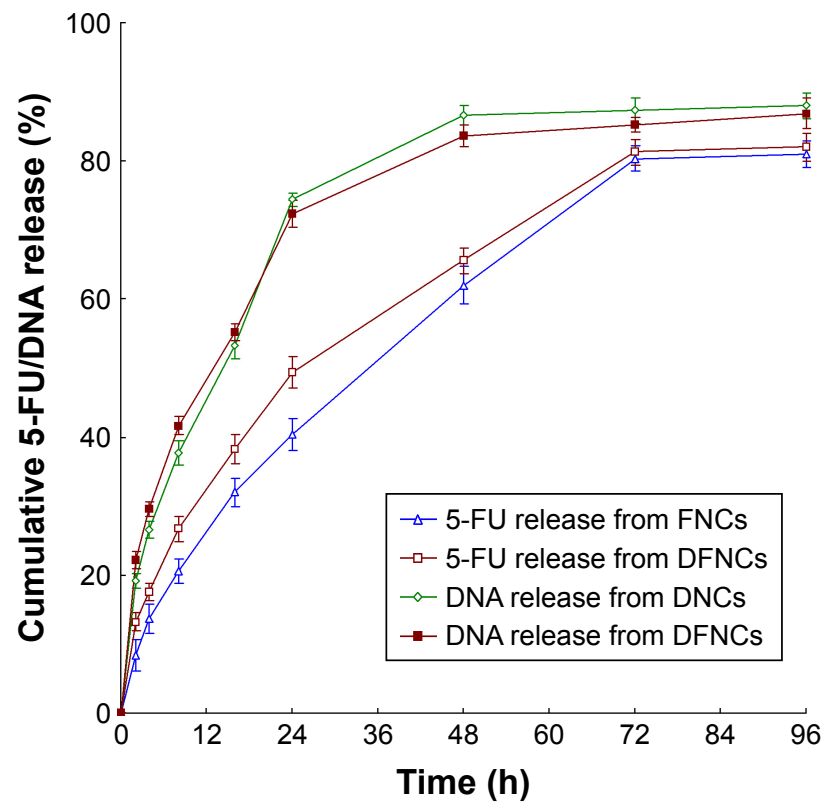

Figure 3 Cumulative 5-FU and DNA release profiles of FNCs, DNCs, and DFNCs.

Note: Data are presented as means \pm standard error, $n=3$.

Abbreviations: 5-FU, 5-fluorouracil; DFNC, DNAand 5-FU co-loaded nanocarrier; DNC, DNA-loaded nanocarrier; ENC, empty nanocarrier; FNC, 5-FU-loaded nanocarrier. of the gene transfection efficiency. DNC and DFNC groups showed remarkable more fluorescence cells in the images than the other groups. More fluorescence cells were found in the images at $72 \mathrm{~h}$ than $24 \mathrm{~h}$. ENCs and FNCs had no fluorescence in the images. Naked pEGFP revealed very weak fluorescence that is almost negligible. The quantitative results confirmed the qualitative results (Figure 5B). DNC and DFNC groups showed over $30 \%$ and nearly $60 \%$ of pEGFP-positive cells in 24 and 72 hours, respectively. On the contrary, other groups did not get obvious transfection results. pEGFP-loaded NCs displayed remarkably higher transfection efficiency than naked DNA $(P<0.05)$.

\section{In vivo anticancer activity}

The tumor growth curves of each group are presented in Figure 6A. The results indicated that treatment with FNCs and DFNCs showed profound suppressed tumor growth than that of free 5-FU $(P<0.05)$. The tumor volume of FNCs and DFNCs at day 21 was 334 and $316 \mathrm{~mm}^{3}$, which is significantly smaller compared with free 5-FU group $\left(852 \mathrm{~mm}^{3}\right)$. Body weights of mice were slightly increased with time in the FNCs and DFNCs groups, while the free drug, DNCs, ENCs, and control groups showed decreases in body weight (Figure 6B). Tumor inhibition ratios of NCs and free 5-FU were summarized in Table 2.

\section{In vivo pharmacokinetics}

After injection of 5-FU or the 5-FU-loaded NCs, plasma levels of 5-FU were determined at specific time points. As shown in Figure 7, all the three groups (free 5-FU, FNC, and 
A

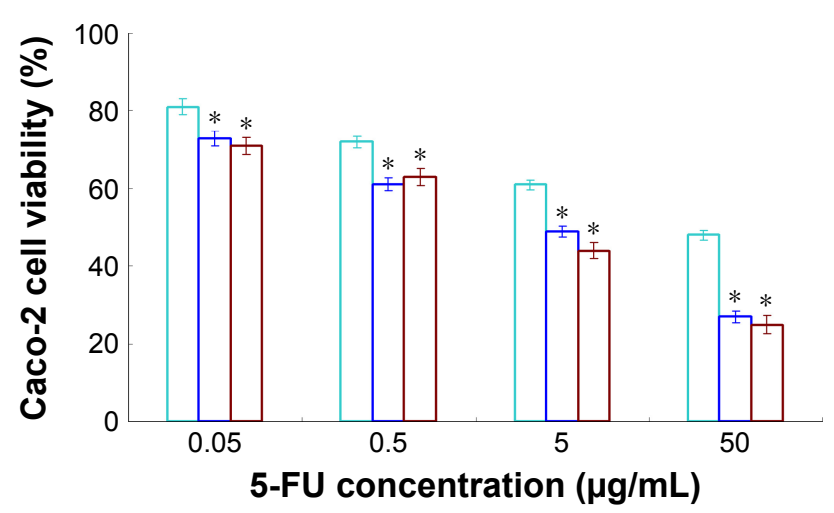

B

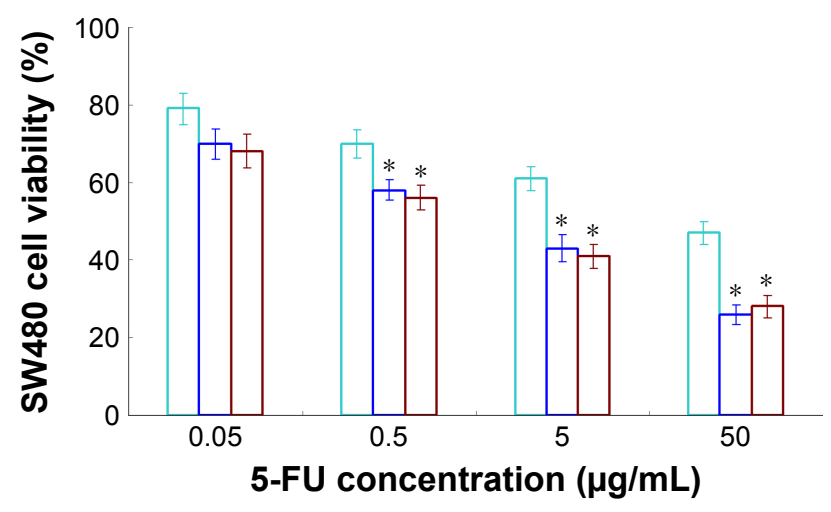

C

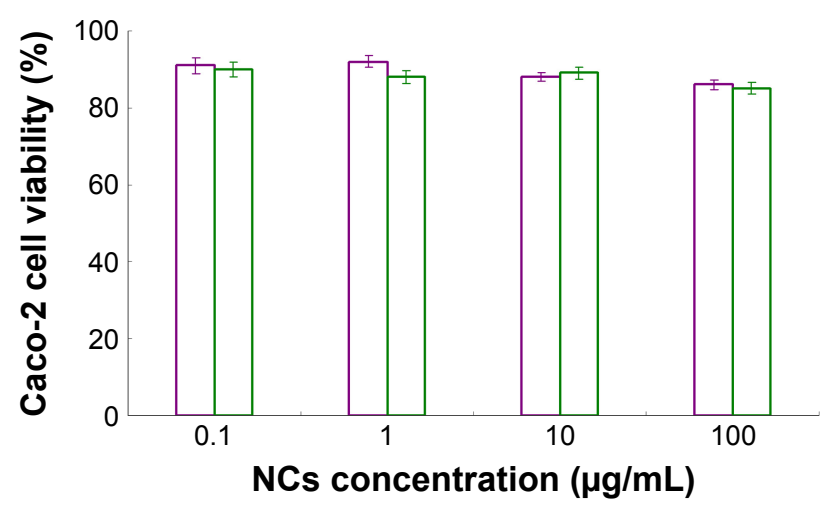

D

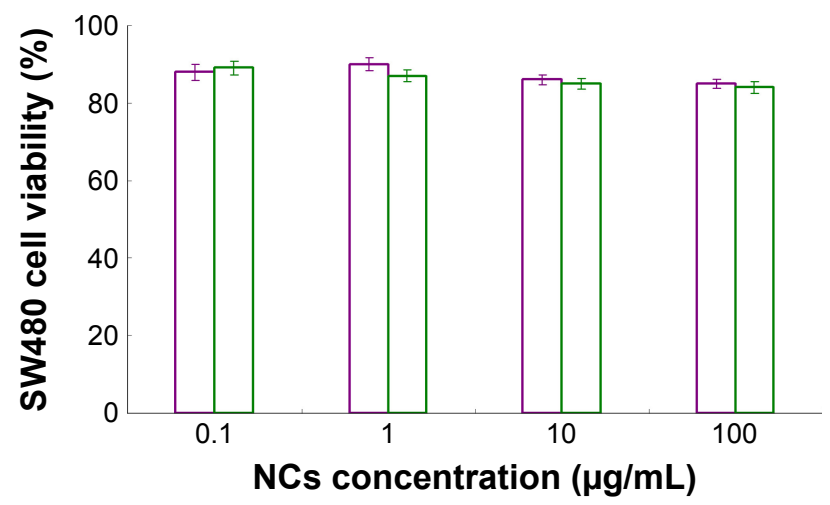

$\square$ ENCs $\square$ DNCs

Figure 4 Cell growth inhibition effects of NCs on Caco-2 and SW480 cells measured by MTT assay.

Notes: (A) 5-FU, FNCs, and DFNCs evaluated on Caco-2; (B) 5-FU, FNCs, and DFNCs evaluated on SW480 cells; (C) ENCs and DNCs evaluated on Caco-2; (D) ENCs and DNCs evaluated on SW480 cells. Data are presented as mean \pm standard error, $n=6$. $* P<0.05$.

Abbreviations: 5-FU, 5-fluorouracil; DFNC, DNA and 5-FU co-loaded nanocarrier; DNC, DNA-loaded nanocarrier; ENC, empty nanocarrier; FNC, 5-FU-loaded nanocarrier; NC, nanocarrier.

DFNC groups) exhibited biphasic behavior with an initial rapid elimination rate followed by a slower drug elimination phase. The maximum 5-FU plasma concentration in FNCs or DFNCs (47 and $49 \mu \mathrm{g} / \mathrm{mL}$, respectively) were significantly greater than free 5-FU $(13 \mu \mathrm{g} / \mathrm{mL})$. At 24 hours, drug level of $\mathrm{NC}$ groups was about $2.8 \mu \mathrm{g} / \mathrm{mL}$ compared to $0.02 \mu \mathrm{g} / \mathrm{mL}$ of free 5-FU. The area under the curve (AUC) of free 5-FU, FNC, and DFNC groups was 17, 284, and $296 \mu \mathrm{g} / \mathrm{mL} \cdot \mathrm{h}$, respectively.

\section{Discussion}

The average hydrodynamic size of ENCs and FNCs had no obvious difference; this means loading of 5-FU had no obvious influence on the hydrodynamic size of NCs. After DNA entrapped into the NCs, the size showed a remarkable increase. This could be explained by the DNA located in the outer layer of NCs, thus enlarging the particle size. The zeta potential of blank NCs was positive; DNA entrapment did decrease the surface charge remarkably. Considered along with the size changes, the DNA was loaded to the NC system by electrostatic absorption with the cationic carriers, causing the increase in size and neutralization of the surface charge. To better protect the gene against enzymatic degradation and achieve higher gene expression in vivo, higher DNA binding ability of NCs is expected. ${ }^{34}$ PicoGreen fluorometry method was applied to determine the GEE of NCs. The GEE of NCs was $\sim 90 \%$, suggesting good DNA-loading ability of NCs. The high DEE of the NCs indicated that DNA binding did not detach the drug from the carriers and that the systems are stable. ${ }^{35}$

The stability of developed NCs was tested in serumincluded media. NCs exhibited no obvious changes in the 
A
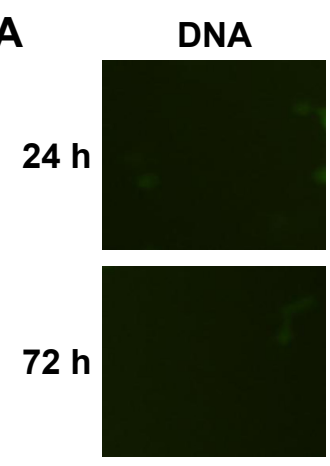

\section{ENCs}
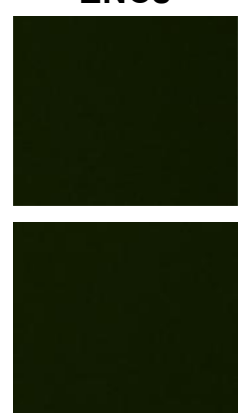

FNCs
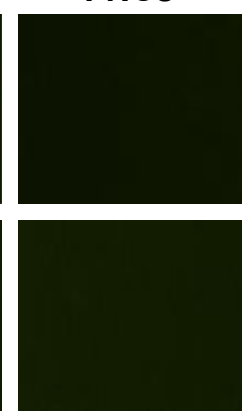

\section{DNCs}
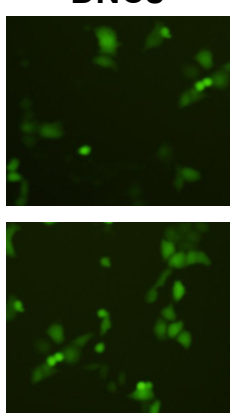

\section{DFNCs}
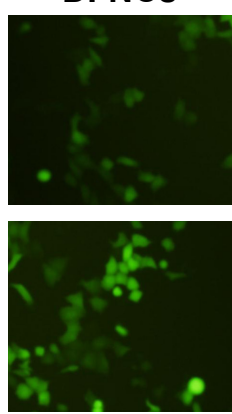

B

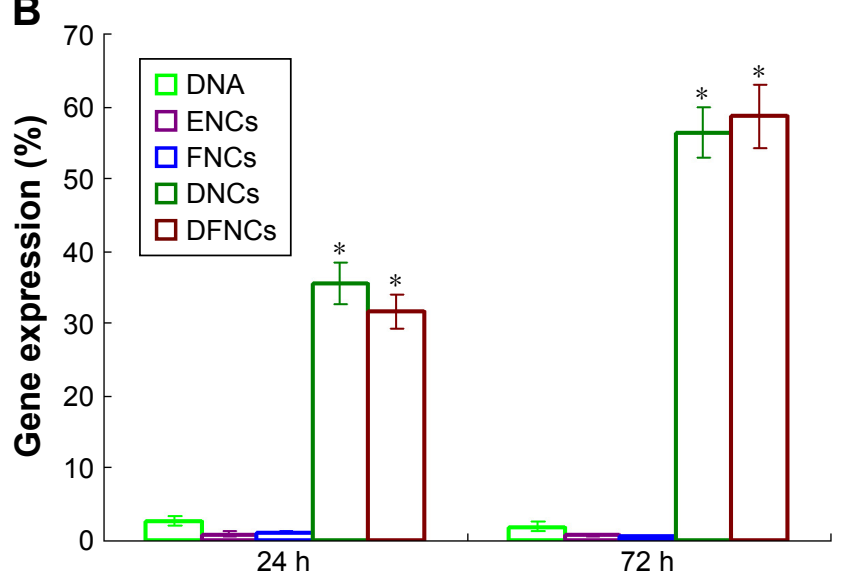

Figure 5 In vivo gene transfection activity of NCs evaluated by fluorescent images $(\mathbf{A})$ with a camera adapter magnification of 0.63 and flow cytometry (B). Notes: Data are presented as means \pm standard error, $n=6$. $* P<0.05$.

Abbreviations: 5-FU, 5-fluorouracil; DFNC, DNA and 5-FU co-loaded nanocarrier; DNC, DNA-loaded nanocarrier; ENC, empty nanocarrier; FNC, 5-FU-loaded nanocarrier; NC, nanocarrier.
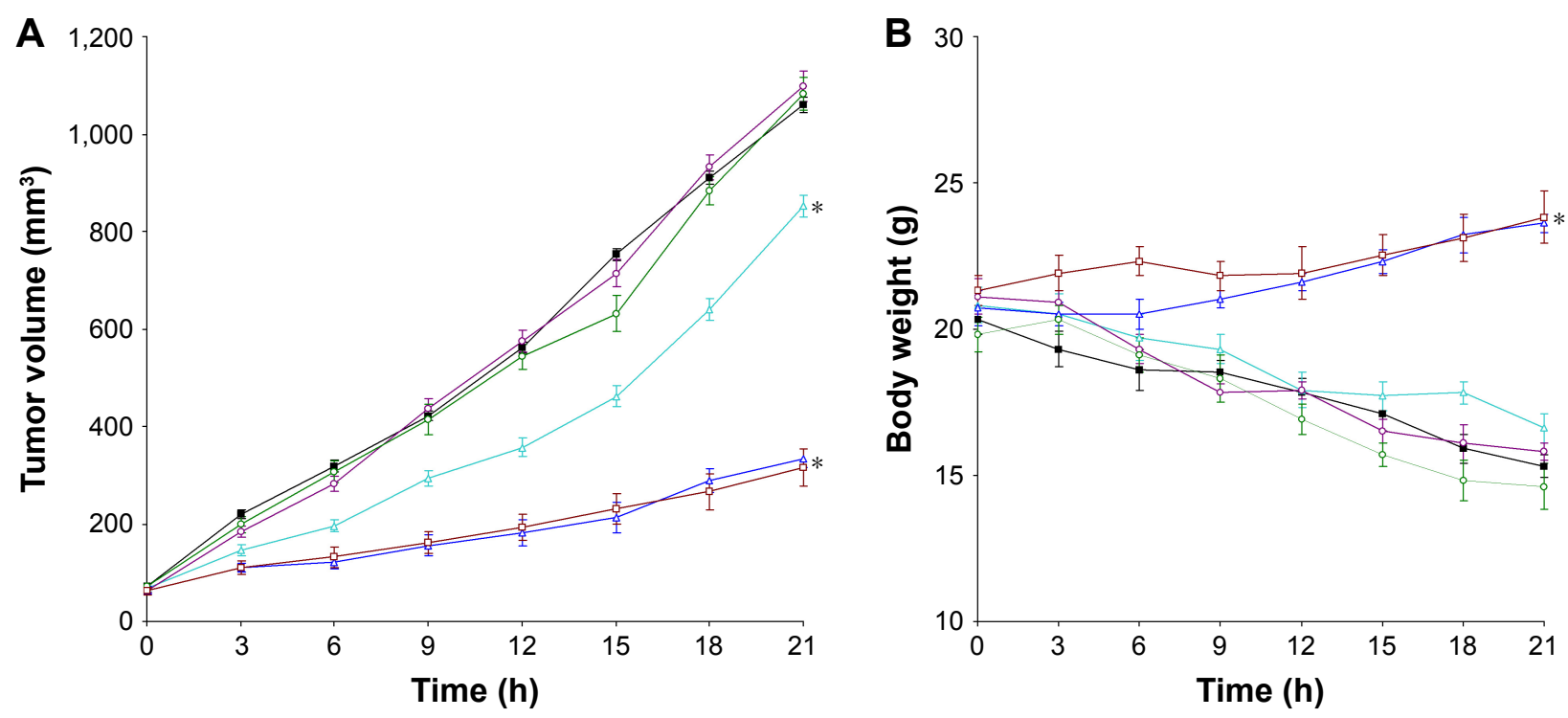

$\rightarrow-$ Blank control $\rightarrow 5-F U \rightarrow$ ENCs $\rightarrow$ FNCs $\multimap$ DNCs $\rightarrow$ DFNCs

Figure 6 In vivo antitumor efficiency evaluated by tumor volume $(\mathbf{A})$ and body weight (B).

Notes: Data are presented as means \pm standard error, $n=8$. $* P<0.05$.

Abbreviations: 5-FU, 5-fluorouracil; DFNC, DNA and 5-FU co-loaded nanocarrier; DNC, DNA-loaded nanocarrier; ENC, empty nanocarrier; FNC, 5-FU-loaded nanocarrier; NC, nanocarrier. 
Table 2 Tumor inhibition ratios of NCs and free 5-FU (mean \pm standard error, $\mathrm{n}=8$ )

\begin{tabular}{llll}
\hline Systems & 5-FU & FNCs & DFNCs \\
\hline Tumor inhibition ratios (\%) & $22.5 \pm 1.9$ & $69.6 \pm 3.6$ & $71.3 \pm 3.8$
\end{tabular}

Abbreviations: 5-FU, 5-fluorouracil; DFNC, DNA and 5-FU co-loaded nanocarrier; FNC, 5-FU-loaded nanocarrier; NC, nanocarrier.

hydrodynamic size after mixing with serum media. ${ }^{36}$ This may contribute to the maintenance of colloidal stability even in serum-included media. The reason for not having a protein corona for these NCs may be the PEG chains presented on the $\mathrm{NC}$ surface that keep the NCs from the coating of protein in the serum. The release profiles of 5-FU and DNA from NCs are different. The release of DNA from NCs was faster than 5-FU, which may be because the DNA was located on the outer layer of NCs and thus could be released earlier. The release of drug from FNCs was a bit faster than that from DFNCs. This could be attributed to the DNA binding that hindered the drug release until most of the DNA left from the carriers. 5-FU released from DFNCs was slower at the first 24 hours because the DNA was located on the surface of the NCs.

In vitro antiproliferation activity of NCs was tested in Caco-2 and SW480 cells. Whether the cytotoxicity of the NCs will be retained during a therapeutic period is of vital importance. ${ }^{37}$ The cell viability of the treated cells was

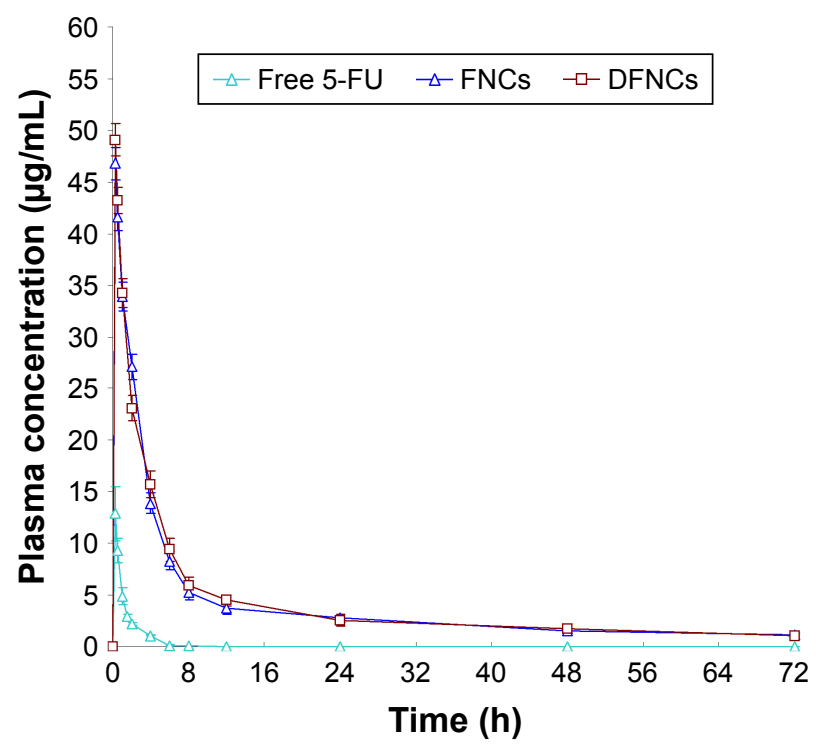

Figure 7 In vivo plasma drug concentration profiles of free 5-FU, FNCs, and DFNCs.

Notes: Data are presented as means \pm standard error, $n=8$.

Abbreviations: 5-FU, 5-fluorouracil; DFNC, DNA and 5-FU co-loaded nanocarrier; FNC, 5-FU-loaded nanocarrier; NC, nanocarrier. calculated for 3 days. The results showed that ENCs and DNA-loaded NCs (without drug) showed no obvious cytotoxicity, which could be the evidence of the low toxicity of the pEGFP and the materials used in the preparation. 5-FUcontaining groups showed dose-dependent cytotoxicity. Significant improvement in cell inhibition efficiency was achieved by 5 -FU-loaded NCs than free 5-FU. These results are in accordance with the research of Hamidreza Kheiri et al that the bare nanoparticles showed little inherent cytotoxicity whereas the statin-loaded nanoparticles were cytotoxic on cancer cells. ${ }^{38}$ The gene delivery ability of NCs to tumor was analyzed in vivo. DNC and DFNC groups exhibited higher gene transfection efficiency than pEGFP and other groups at all time points. More fluorescence cells were found in the images at $72 \mathrm{~h}$ than $24 \mathrm{~h}$. Considering the sustained release manner of the NCs, we could further ensure the better gene transfection after a longer time (72 hours compared to 24 hours) of administration. The qualitative and quantitative results suggested the in vivo efficiency of the NCs for colon cancer gene therapy. Nanoparticles could have the potential to improve the expression of genes was also reported by Saghebasl et al in their study. ${ }^{39}$

The antitumor efficacy of NCs was further examined in tumor-bearing mice. The tumor growth rate was not found to significantly decrease with free 5-FU and 5-FU-loaded NC treatment. The tumor growth rate was significantly decreased in the group treated with FNCs and DFNCs than that of free 5-FU. Dai et al also introduced a block copolymer to treat colon carcinoma. Compared with control group, a significant decrease in the number of tumor nodes was observed in group treated with drug-loaded nanoparticles. The results are similar with our present research. ${ }^{40}$ In vivo pharmacokinetic study showed the biphasic elimination behaviors of the 5-FUloaded NCs. ${ }^{41,42}$ The fact that the drug-loaded NCs follow a biphasic elimination pattern is consistent with data suggesting that a large proportion of the encapsulated 5-FU is lost from the NCs following administration. The second phase of elimination may be due to a fraction of the 5-FU that remains more tightly associated with the inner core of the NCs.

\section{Conclusion}

These results indicate that drug and gene co-loaded NCs have improved antitumor effects and an excellent gene delivery efficiency to the tumor site. Therefore, DFNCs can be used as a promising nanomedicine for the delivery of antitumor drugs and genes and may significantly contribute to colorectal cancer therapy. 


\section{Disclosure}

The authors report no conflicts of interest in this work.

\section{References}

1. Siegel RL, Miller KD, Jemal A. Cancer statistics, 2017. CA Cancer J Clin. 2017;67(1):7-30.

2. Siegel RL, Miller KD, Fedewa SA, et al. Colorectal cancer statistics, 2017. CA Cancer J Clin. 2017;67(3):177-193.

3. Marquez J, Fernandez-Piñeiro I, Araúzo-Bravo MJ, et al. Targeting liver sinusoidal endothelial cells with miR-20a-loaded nanoparticles reduces murine colon cancer metastasis to the liver. Int J Cancer. 2018; 143(3):709-719.

4. Serini S, Cassano R, Corsetto PA, Rizzo AM, Calviello G, Trombino S. Omega-3 PUFA loaded in resveratrol-based solid lipid nanoparticles: physicochemical properties and antineoplastic activities in human colorectal cancer cells in vitro. Int J Mol Sci. 2018; 19(2):586.

5. Lee CS, Ryan EJ, Doherty GA. Gastro-intestinal toxicity of chemotherapeutics in colorectal cancer: the role of inflammation. World $J$ Gastroenterol. 2014;20(14):3751-3761.

6. Kozovska Z, Gabrisova V, Kucerova L. Colon cancer: cancer stem cells markers, drug resistance and treatment. Biomed Pharmacother. 2014;68(8):911-916.

7. Rosman R, Saifullah B, Maniam S, Dorniani D, Hussein MZ, Fakurazi S. Improved anticancer effect of magnetite nanocomposite formulation of GALLIC acid $\left(\mathrm{Fe}_{3} \mathrm{O}_{4}\right.$-PEG-GA) against lung, breast and colon cancer cells. Nanomaterials. 2018;8(2):E83.

8. Li L, Deng R, Su Y, Yang C. Dual-targeting nanoparticles with excellent gene transfection efficiency for gene therapy of peritoneal metastasis of colorectal cancer. Oncotarget. 2017;8(52):89837-89847.

9. Yang H, Choi HJ, Park SH, Kim JS, Moon Y. Macrophage inhibitory cytokine-1 (MIC-1) and subsequent urokinase-type plasminogen activator mediate cell death responses by ribotoxic anisomycin in HCT-116 colon cancer cells. Biochem Pharmacol. 2009;78(9):1205-1213.

10. du J, Li L. Which one performs better for targeted lung cancer combination therapy: pre- or post-bombesin-decorated nanostructured lipid carriers? Drug Deliv. 2016;23(5):1799-1809.

11. Shao Z, Shao J, Tan B, et al. Targeted lung cancer therapy: preparation and optimization of transferrin-decorated nanostructured lipid carriers as novel nanomedicine for co-delivery of anticancer drugs and DNA. Int J Nanomedicine. 2015;10:1223-1233.

12. Dong S, Zhou X, Yang J. TAT modified and lipid - PEI hybrid nanoparticles for co-delivery of docetaxel and pDNA. Biomed Pharmacother. 2016;84:954-961.

13. Baoum AA, Middaugh CR, Berkland C. DNA complexed with TAT peptide and condensed using calcium possesses unique structural features compared to PEI polyplexes. Int J Pharm. 2014;465(1-2):11-17.

14. Piña MJ, Girotti A, Santos M, Rodríguez-Cabello JC, Arias FJ. Biocompatible ELR-based polyplexes coated with MUC1 specific aptamers and targeted for breast cancer gene therapy. Mol Pharm. 2016;13(3):795-808.

15. Tsien RY. The green fluorescent protein. Annu Rev Biochem. 1998;67: 509-544.

16. Yang TT, Cheng L, Kain SR. Optimized codon usage and chromophore mutations provide enhanced sensitivity with the green fluorescent protein. Nucleic Acids Res. 1996;24(22):4592-4593.

17. Arpino JA, Rizkallah PJ, Jones DD. Crystal structure of enhanced green fluorescent protein to $1.35 \AA$ resolution reveals alternative conformations for Glu222. PLoS One. 2012;7(10):e47132.

18. Kong F, Zhou F, Ge L, Liu X, Wang Y. Mannosylated liposomes for targeted gene delivery. Int J Nanomedicine. 2012;7:1079-1089.

19. Yu W, Liu C, Ye J, Zou W, Zhang N, Xu W. Novel cationic SLN containing a synthesized single-tailed lipid as a modifier for gene delivery. Nanotechnology. 2009;20(21):215102.
20. Jorge AF, Aviñó A, Pais A, Eritja R, Fàbrega C. DNA-based nanoscaffolds as vehicles for 5-fluoro-2'-deoxyuridine oligomers in colorectal cancer therapy. Nanoscale. 2018;10(15):7238-7249.

21. Kamel KM, Khalil IA, Rateb ME, Elgendy H, Elhawary S. ChitosanCoated Cinnamon/Oregano-Loaded Solid Lipid Nanoparticles to Augment 5-Fluorouracil Cytotoxicity for Colorectal Cancer: Extract Standardization, Nanoparticle Optimization, and Cytotoxicity Evaluation. J Agric Food Chem. 2017;65(36):7966-7981.

22. Pretel E, Arias JL, Cabeza L, et al. Development of biomedical 5-fluorouracil nanoplatforms for colon cancer chemotherapy: Influence of process and formulation parameters. Int J Pharm. 2017;530(1-2):155-164.

23. Sharma A, Kaur A, Jain UK, Chandra R, Madan J. Stealth recombinant human serum albumin nanoparticles conjugating 5-fluorouracil augmented drug delivery and cytotoxicity in human colon cancer, HT-29 cells. Colloids Surf B Biointerfaces. 2017;155:200-208.

24. Rychahou P, Bae Y, Reichel D, et al. Colorectal cancer lung metastasis treatment with polymer-drug nanoparticles. J Control Release. 2018; 275:85-91.

25. Liu C, Liu F, Feng L, Li M, Zhang J, Zhang N. The targeted co-delivery of DNA and doxorubicin to tumor cells via multifunctional PEI-PEG based nanoparticles. Biomaterials. 2013;34(10):2547-2564.

26. Kim S, Lee SY, Cho HJ. Doxorubicin-Wrapped Zinc Oxide Nanoclusters for the Therapy of Colorectal Adenocarcinoma. Nanomaterials. 2017;7(11):E354:354.

27. Gao Z, Li Z, Yan J, Wang P. Irinotecan and 5-fluorouracil-co-loaded, hyaluronic acid-modified layer-by-layer nanoparticles for targeted gastric carcinoma therapy. Drug Des Devel Ther. 2017;11:2595-2604.

28. Han Y, Zhang Y, Li D, Chen Y, Sun J, Kong F. Transferrin-modified nanostructured lipid carriers as multifunctional nanomedicine for codelivery of DNA and doxorubicin. Int J Nanomedicine. 2014;9:4107-4116.

29. Yu W, Liu C, Liu Y, Zhang N, Xu W. Mannan-modified solid lipid nanoparticles for targeted gene delivery to alveolar macrophages. Pharm Res. 2010;27(8):1584-1596.

30. Ibarra LE, Porcal GV, Macor LP, et al. Metallated porphyrin-doped conjugated polymer nanoparticles for efficient photodynamic therapy of brain and colorectal tumor cells. Nanomedicine. 2018;13(6): 605-624.

31. Han Y, Li Y, Zhang P, et al. Nanostructured lipid carriers as novel drug delivery system for lung cancer gene therapy. Pharm Dev Technol. 2016; 21(3):277-281.

32. Han Y, Zhang P, Chen Y, Sun J, Kong F. Co-delivery of plasmid DNA and doxorubicin by solid lipid nanoparticles for lung cancer therapy. Int J Mol Med. 2014;34(1):191-196.

33. Thomas AM, Kapanen AI, Hare JI, et al. Development of a liposomal nanoparticle formulation of 5-fluorouracil for parenteral administration: formulation design, pharmacokinetics and efficacy. $J$ Control Release. 2011;150(2):212-219.

34. Kaur R, Chitanda JM, Michel D, et al. Lysine-functionalized nanodiamonds: synthesis, physiochemical characterization, and nucleic acid binding studies. Int J Nanomedicine. 2012;7:3851-3866.

35. Rahman HS, Rasedee A, How CW, et al. Zerumbone-loaded nanostructured lipid carriers: preparation, characterization, and antileukemic effect. Int J Nanomedicine. 2013;8:2769-2781.

36. Dong T, Chen N, Ma X, et al. The protective roles of L-borneolum, D-borneolum and synthetic borneol in cerebral ischaemia via modulation of the neurovascular unit. Biomed Pharmacother. 2018;102:874-883.

37. Miao JF, Peng YF, Chen S, et al. A novel harmine derivative, N-(4(hydroxycarbamoyl)benzyl)-1-(4-methoxyphenyl)-9H-pyrido[3,4-b] indole-3-carboxamide (HBC), as histone deacetylase inhibitor: in vitro antiproliferation, apoptosis induction, cell cycle arrest, and antimetastatic effects. Eur J Pharmacol. 2018;824:78-88.

38. Hamidreza Kheiri M, Alimohammadi N, Danafar H. Preparation of biocompatible copolymeric micelles as a carrier of atorvastatin and rosuvastatin for potential anticancer activity study. Pharm Dev Technol.2018:1-11. In press. 
39. Saghebasl S, Davaran S, Rahbarghazi R, Montaseri A, Salehi R, Ramazani A. Synthesis and in vitro evaluation of thermosensitive hydrogel scaffolds based on (PNIPAAm-PCL-PEG-PCL-PNIPAAm)/ Gelatin and (PCL-PEG-PCL)/Gelatin for use in cartilage tissue engineering. J Biomater Sci Polym Ed. 2018;29(10):1185-1206.

40. Dai M, Xu X, Song J, et al. Preparation of camptothecin-loaded PCEC microspheres for the treatment of colorectal peritoneal carcinomatosis and tumor growth in mice. Cancer Lett. 2011;312(2):189-196.
41. Doodnaught GM, Monteiro BP, Benito J, et al. Pharmacokinetic and pharmacodynamic modelling after subcutaneous, intravenous and buccal administration of a high-concentration formulation of buprenorphine in conscious cats. PLoS One. 2017;12(4):e0176443.

42. Xie M, Fan D, Li Y, et al. Supercritical carbon dioxide-developed silk fibroin nanoplatform for smart colon cancer therapy. Int J Nanomedicine. 2017;12:7751-7761

\section{Publish your work in this journal}

Drug Design, Development and Therapy is an international, peerreviewed open-access journal that spans the spectrum of drug design and development through to clinical applications. Clinical outcomes, patient safety, and programs for the development and effective, safe, and sustained use of medicines are the features of the journal, which has also been accepted for indexing on PubMed Central. The manuscript management system is completely online and includes a very quick and fair peer-review system, which is all easy to use. Visit http://www.dovepress.com/testimonials.php to read real quotes from published authors.

Submit your manuscript here: http://www.dovepress.com/drug-design-development-and-therapy-journal 